\title{
Escolha de parceiros sexuais em Anastrepha zenildae (Zucchi, 1979, Diptera: Tephritidae): papel dos caracteres morfológicos
}

\author{
Lucia Maria de Almeida * \\ Liana Lucas Rocha \\ Midiam Lima de Souza \\ Norma Helena Duarte Mendes \\ João Maria Gomes Alencar de Souza \\ Faculdade de Ciências, Cultura e Extensão do Rio Grande do Norte \\ Rua Orlando Silva, 2897, Capim Macio, CEP 59.080-020, Natal - RN, Brasil \\ * Autor para correspondência \\ lalmeida19@yahoo.com.br
}

Submetido em 11/04/2012

Aceito para publicação em 18/03/2013

\section{Resumo}

Machos de Anastrepha sexualmente ativos formam grupos e exibem comportamento de corte para atrair as fêmeas, formação típica da estratégia de lek. As fêmeas escolhem um macho para copular, provavelmente utilizando parâmetros morfológicos e comportamentais. Este estudo teve por objetivo identificar o papel dos caracteres morfológicos na seleção sexual de A. zenildae. As observações do comportamento de cópula foram realizadas em unidades experimentais (16 machos e 16 fêmeas virgens) durante a fotofase por três dias consecutivos. Machos que acasalaram foram marcados. Para as análises morfométricas dos machos foram utilizados cinco caracteres: comprimento da asa direita, comprimento do tórax, largura da cabeça, largura da face e número de cerdas da placa frontal. As medidas foram feitas a partir das imagens capturadas utilizando o programa Motic Imagens Plus 2.0. Para a maioria das características, os machos que obtiveram sucesso de cópula apresentaram valores médios mais elevados do que aqueles que não obtiveram sucesso. As variáveis comprimento do tórax, comprimento de asa e o módulo da diferença entre cerdas da placa frontal mostram-se alvos da seleção sexual, sugerindo que fêmeas podem discriminar os machos por tamanho durante a interação que ocorre na estratégia de lek.

Palavras-chave: Assimetria flutuante; Caracteres morfométricos; Cerdas frontais; Seleção sexual

\section{Abstract}

Choice of sexual partners in Anastrepha zenildae (Zucchi, 1979, Diptera: Tephritidae): role of the morphological traits. Sexually active Anastrepha males form groups and display courting behavior to attract females, a typical form of the lek strategy. Females choose a male to copulate with, perhaps using morphological and behavioral parameters. This study aimed to identify the role of morphological traits on the sexual selection of $A$. zenildae. Observations on the copulation behavioral were conducted at experimental units (16 virgin males and 16 virgin females) during the photophase for three consecutive days. The males which mated were marked. Five traits were used for morphometric analysis of males: right wing length, thorax length, head width, face 
width, and number of bristles on the front plate. The measurements were made through images captured using the software Motic Images Plus 2.0. For most traits, the males which were successful regarding copulation showed higher mean values than those which weren't successful. The variables thorax length, wing length, and the difference module between front plate bristles show to be sexual selection targets, suggesting that females can discriminate males by size during the interaction occurring in the lek strategy.

Key words: Fluctuating asymmetry; Frontal bristles; Morphometric traits; Sexual selection

\section{Introdução}

A aplicação dos conhecimentos sobre a seleção sexual em espécies de moscas-das-frutas do gênero Anastrepha pode auxiliar os programas de manejo, monitoramento e controle populacional destes insetos praga. A seleção sexual abrange a competição entre os machos ao acesso às fêmeas e a escolha da fêmea por um determinado parceiro, e consequentemente a sua constância e permanência dentro de um pomar ou área natural (THORNHILL, 1992).

Alguns estudos mostraram que a melhor estratégia para machos é frequentemente acasalar com o maior número de fêmeas possível, enquanto que para fêmeas a melhor estratégia é selecionar o macho mais atrativo (GWYNNE, 1984; PIZZARI, 2003). A escolha do parceiro por fêmeas foi evidenciada em muitas espécies (ANDERSSON, 1994; JENNIONS; PETRIE, 1997) e os sinais mais importantes usados no reconhecimento para o acasalamento são baseados em uma avaliação de características visuais, acústicas ou químicas do fenótipo masculino (LUCK; JOLY 2005).

No gênero Anastrepha o comportamento sexual apresenta dois tipos de estratégias: a cópula forçada e formação de lek. As espécies monófagas e univoltinas empregam a cópula forçada, onde o macho estabelece e defende um território em uma planta hospedeira contendo frutos suscetíveis a oviposição, e quando a fêmea o invade, procurando por sítios de oviposição, ele força a cópula (SIVINSKI; BURK, 1989). A espécie $A$. bistrigata é a única na qual foi observado este tipo de estratégia (SELIVON et al., 1991).

A estratégia de formação de lek é adotada pelas espécies polífagas e multivoltinas, na qual um macho emite sinais que atraem outros machos para um local de exibição comunitária e iniciam elaboradas sequências de comportamentos de chamada, envolvendo liberação de feromônio sexual e vibração das asas com produção de sons, atraindo fêmeas sexualmente receptivas. Após interagir com vários machos, a fêmea é capaz de discriminar e copular com apenas um, geralmente o encontro ocorre nas folhas da planta hospedeira (PROKOPY, 1980). De acordo com Emlen e Oring (1977), leks são agregações de machos que as fêmeas visitam somente com o propósito de acasalar. Aluja et al. (2000) definiram lek para espécies do gênero Anastrepha como sendo uma agregação de pelo menos três machos em uma área claramente definida e exibindo comportamento de chamada simultaneamente.

No processo de escolha sexual, a competição entre machos por acesso as fêmeas apresenta uma grande tendência de machos maiores ganharem a disputa (RODRIGUERO et al., 2002b). Enquanto que, no processo de escolha sexual exercido pela fêmea, observase uma preferência por machos maiores e simétricos que são supostamente mais atraentes, indicando que algumas características morfológicas proporcionem uma vantagem seletiva aos seus portadores, conduzindoos a um sucesso reprodutivo significativo (BURK; WEBB, 1983; MOLLER; POMIANKOWSKI, 1993; SCIURANO et al., 2007).

A morfometria tem sido utilizada para quantificar variações na forma e no tamanho das estruturas (DALY, 1985). Estudos envolvendo morfometria têm sido bastante utilizados em sistemática, evolução e seleção sexual em muitos grupos de insetos (ATCHLEY; CHENEY, 1974). Assimetria flutuante é um método de análise morfológica que descreve pequenas diferenças casuais entre caracteres com distribuição bilateral. A assimetria flutuante reflete a ocorrência de ruídos no desenvolvimento dos organismos causados por estresse genético ou ambiental (CLARKE; MCKENZIE, 1992; SOUZA et al., 2007). Estudos recentes envolvendo a 
assimetria flutuante têm despertado muitos interesses devido ao seu papel na seleção sexual de insetos, inclusive das moscas-das-frutas (THORNHILL, 1992; HUNT et al., 1998).

No caso do gênero Anastrepha, existem poucos estudos que enfatizam quais as características morfológicas relacionadas com o sucesso de acasalamento de machos dentro das populações (BURK; WEBB, 1983; SCIURANO et al., 2007). Estudos que investiguem os fatores que determinam a seleção sexual focalizando o papel das características morfológicas são de grande importância no monitoramento da qualidade na produção em massa dos insetos produzidos em biofabricas para utilização em programas de controle de pragas. Rotineiramente, essas biofábricas monitoram a qualidade dos insetos que produzem utilizando como indicadores de qualidade: fecundidade, viabilidade dos ovos, pesos das larvas e pupas, capacidade de voou e competitividade reprodutiva. Esses são procedimentos altamente específicos e laboriosos quando não apresentam baixa sensibilidade para detectar sinais sutis de estresse causado pela alta densidade em que esses insetos são produzidos. Nesse sentido, análises da morfologia podem ser empregadas para avaliar a qualidade dos insetos produzidos e também identificar sinais de deterioração, através de medidas morfometricas (HUNT et al., 1998).

Dentre as várias espécies de moscas-das-frutas, Anastrepha zenildae que tinha sua distribuição geográfica restrita a alguns Estados do nordeste brasileiro, vem ampliando sua distribuição e frequência populacional bem como a quantidade de hospedeiros utilizados (ARAÚJO; ZUCCHI, 2003). Essa espécie tem se tornado potencialmente danosa tanto em pomares domésticos quanto em pomares comerciais (ARAÚJO et al., 2005). Os programas de monitoramento de Anastrepha utilizam substâncias químicas, como feromônios, atrativo em armadilhas para capturar adultos de modo a monitorar a população e a técnica do macho estéril, na tentativa de interferir na reprodução. O sucesso dessas técnicas depende do conhecimento do comportamento reprodutivo natural da espécie praga (CALKINS; ASHLEY, 1989). O objetivo deste estudo foi verificar o papel dos caracteres morfológicos na seleção sexual de Anastrepha zenildae.

\section{Material e Métodos}

\section{Obtenção dos insetos}

Os insetos utilizados neste estudo foram obtidos a partir de colônias existentes no Laboratório de Biologia Evolutiva de insetos pragas do Departamento de Biologia Celular e Genética da Universidade Federal do Rio grande do Norte. As populações foram mantidas em sistema de gerações descontínuas, formadas por linhagens de laboratório criadas em massa e indivíduos selvagens introduzidos a cada geração para evitar o efeito da endogamia. Foi utilizado o mamão (Carica papaya) como substrato para oviposição das fêmeas e desenvolvimento larval. Após a eclosão, os adultos foram separados por sexo e transferidos para caixas de acrílico de 30×30×30 cm, providos de água e dieta artificial para adultos à base de hidrolisado de proteína, Sustagen $\AA$, mel de abelha, açúcar mascavo, extrato de levedura e açúcar refinado fornecidos ad libitum.

Os animais foram mantidos em condições controladas de temperatura a $23 \pm 2{ }^{\circ} \mathrm{C}$, umidade de 80 $\pm 5 \%$ e iluminação artificial. O regime de iluminação foi organizado em ciclo claro/escuro $12 \mathrm{~h} 12 \mathrm{~min}$, com intensidade de 750lux (2 lâmpadas fluorescentes 32W) na fase de claro e abaixo de 1lux na fase de escuro (lâmpada incandescente vermelha de $25 \mathrm{~W}$ ), estando as luzes acesas entre $07 \mathrm{~h} 00 \mathrm{~min}$ e $19 \mathrm{~h} 00 \mathrm{~min}$.

\section{Observações do comportamento de cópula}

A partir da população inicial, foram retirados machos e fêmeas ao acaso para a formação das unidades experimentais, totalizando seis unidades. Essas unidades experimentais foram compostas por 16 machos e 16 fêmeas, todos adultos e virgens com a idade de $20 \pm 1$ dias. Cada unidade experimental constava de uma gaiola de acrílico 30×30×30 cm nas condições descritas acima. Os machos foram colocados uma hora após o acender das luzes e, após $40 \mathrm{~min}$, as fêmeas foram liberadas. Os registros foram realizados de modo continuo a partir do momento de liberação das fêmeas até o apagar das luzes 19 h 00 min, os comportamentos de corte e cópula foram registrados de acordo com a técnica de todas 
as ocorrências (MARTIN; BATESON, 1994). A cada cópula observada, o macho foi retirado e marcado no tórax com tinta à base de água para diferenciá-lo dos machos não aceitos, e em seguida eram recolocados na gaiola de acrílico. Cada unidade experimental foi observada durante três dias consecutivos.

\section{Análises morfométricas}

Para as análises morfométricas, foram utilizados cinco caracteres morfológicos: comprimento da asa direita, comprimento do tórax, largura da cabeça e largura da face de acordo com Rodriguero et al. (2002a) e contagem das cerdas da placa frontal. A assimetria das cerdas frontais foi estimada a partir do módulo da diferença entre o número de cerdas dos lados direito e esquerdo segundo Souza et al. (2007).

As asas foram seccionadas e montadas entre lâmina e lamínula usando bálsamo do Canadá como fixador. Posteriormente, todas as estruturas foram fotografadas utilizando-se microcâmera digital instalada em estéreo microscópio MOTICAM 480 AD (aumento de 8x), acoplado a um computador. As medidas foram feitas em milímetros $(\mathrm{mm})$, a partir das imagens capturadas utilizando-se o programa Motic Imagens Plus 2.0. A contagem das cerdas da placa frontal foi realizada utilizando-se o mesmo sistema de captura de imagens anteriormente citado.

\section{Análise dos dados}

No presente estudo, executou-se uma regressão logística múltipla com o objetivo de relacionar as cinco características descritas acima com o sucesso de cópula para calcular a relação de probabilidade de sucesso de cópula para indivíduos portadores de determinadas medidas de algumas partes do corpo. Utilizamos o teste do qui-quadrado $\chi^{2}$ para comparar o sucesso de cópula com a assimetria ou simetria de cerdas da placa frontal. Realizamos também o teste de correlação de Pearson entre as cinco características. Para todos os testes consideramos significantes os valores de $p \leq 0,05$ bicaudal. Os dados foram analisados usando o software Statistica ${ }^{\circledR} 5.5$.

\section{Resultados}

Observamos que o comportamento sexual de A. zenildae compreende duas fases distintas: a corte (comportamento de chamada, correspondendo à vibração das asas e liberação de feromônio) e a cópula. Durante o comportamento de chamada, os machos são atraídos por sinais sexuais de outros machos, formando-se um agrupamento de machos $(6,0 \pm 1,0$ machos) que apresentam esses sinais simultaneamente, além de episódios de agressão que sugerem a disputa por território, o que indica a existência da formação de lek. As fêmeas aproximavam-se do lek andando ou com curtos voos, sendo observada intensa atividade locomotora dos machos durante a corte. Um dos machos, geralmente o que estava no centro do lek, orientava-se frontalmente em relação à fêmea, ficando frente a frente a uma distância de aproximadamente $2 \mathrm{a} 4 \mathrm{~cm}$. A postura de ficar frente a frente e tocarem rapidamente cabeça com cabeça pareceram decisivos para que a cópula fosse efetivada, pois quase todas as cópulas observadas aconteceram depois desta etapa.

Da amostra de 97 machos analisados, dos 42 que copularam um total de $59,5 \%$ apresentou simetria com relação às cerdas da placa frontal e 40,5\% eram assimétricos; enquanto dos 55 que não efetivaram cópula, $34,5 \%$ eram simétricos e $65,5 \%$ assimétricos. Quando a assimetria relativa ao número de cerdas da placa frontal e o sucesso de cópula, foram analisadas em conjunto, constatamos uma diferença significativa na interação dessas variáveis $\left(\chi^{2}=7,6 ; p \leq 0,05\right)$ (Figura 1).

Para a maioria das características, os machos que obtiveram sucesso de cópula apresentaram valores médios mais elevados do que os machos que não obtiveram sucesso de cópula (Tabela 1).

A análise de regressão logística múltipla aplicada às cinco variáveis selecionadas revelou que, de acordo com a relação de probabilidade (RP), as características: comprimento do tórax $(\mathrm{RP}=379,3 ; \mathrm{p} \leq 0,001)$, comprimento da asa $(\mathrm{RP}=22,4 ; \mathrm{p}=0,001)$ e o módulo da diferença das cerdas frontais $(\mathrm{RP}=0,2 ; \mathrm{p}=0,015)$ são possíveis alvos da seleção sexual. 
FIGURA 1: Porcentagem de machos de Anastrepha zenildae simétricos e assimétricos no número de cerdas da placa frontal em relação ao sucesso de cópula.

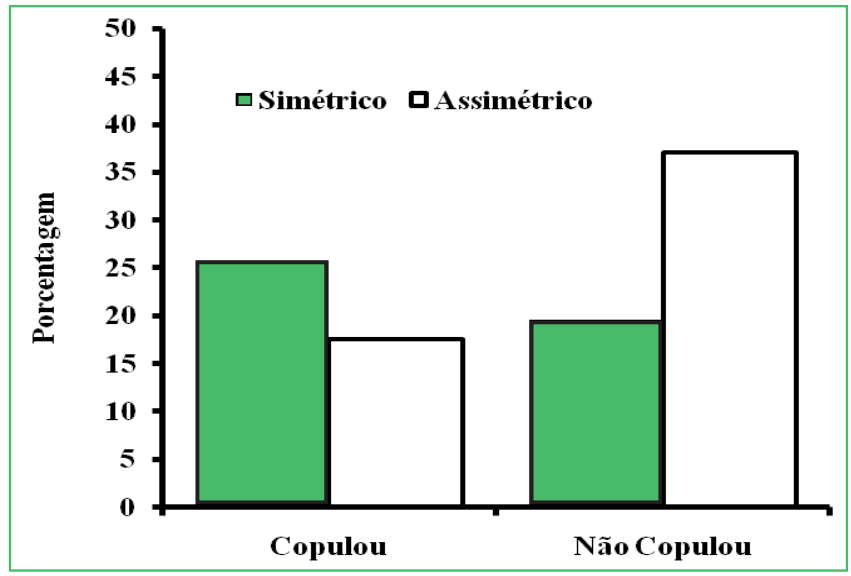

TABELA 1: Medidas (mm, média \pm DP) das características morfológicas de machos de Anastrepha zenildae em função do sucesso de cópula.

\begin{tabular}{lcc}
\hline \multirow{2}{*}{ Características } & \multicolumn{2}{c}{ Machos } \\
\cline { 2 - 3 } & $\begin{array}{c}\text { Com cópula } \\
(\mathbf{n}=\mathbf{4 2})\end{array}$ & $\begin{array}{c}\text { Sem cópula } \\
(\mathbf{n}=\mathbf{5 5})\end{array}$ \\
\hline Comprimento do tórax & $2,94 \pm 0,18$ & $2,78 \pm 0,27$ \\
Largura da cabeça & $2,04 \pm 0,09$ & $1,97 \pm 0,18$ \\
Largura da face & $0,67 \pm 0,06$ & $0,67 \pm 0,07$ \\
Comprimento da asa & $5,18 \pm 0,36$ & $4,98 \pm 0,40$ \\
\hline
\end{tabular}

Contudo, se olharmos essas características em ordem de importância, observa-se que o comprimento do tórax e o comprimento de asa são as principais características, indicando que estruturas maiores favorecem o sucesso de cópula. Por outro lado, o valor do coeficiente $\mathrm{B}$ do módulo da diferença entre as cerdas frontais apresenta-se negativo, o que implica em assimetria não favorecendo o sucesso de cópula (Tabela 2).

As características largura da cabeça e da face apresentaram valores não significativos e somente a primeira apresenta correlação significativa com o comprimento do tórax $(r=0,9 ; p=0,001)$. Levando em conta apenas os caracteres significativos, reanalisamos os dados (Tabela 3) e obtivemos a seguinte equação de regressão logística que prediz a probabilidade de sucesso de cópula (PSC) para machos de $A$. zenildae:

$\mathrm{PSC}=-32,887+6,379(\mathrm{CT})-1,437(\mathrm{CF})+3,025(\mathrm{CA})$

Onde:

$\mathrm{CT}=$ comprimento do tórax;

$\mathrm{CF}=$ módulo da diferença das cerdas frontais;

$\mathrm{CA}=$ comprimento da asa.

TABELA 2: Análise por regressão logística múltipla das cinco características morfométricas de machos de Anastrepha zenildae.

\begin{tabular}{lccccc}
\hline Constante e Características & Coeficiente B & $\begin{array}{c}\text { Erro } \\
\text { Padrão }\end{array}$ & $\begin{array}{c}\text { Relação } \\
\text { Probabilística }\end{array}$ & $\mathbf{z}$ & $\mathbf{p}$ \\
\hline Constante & $-33,677$ & 7,834 & - & $-4,299$ & 0,001 \\
Comprimento do tórax & 5,938 & 2,370 & 379,302 & 2,506 & 0,012 \\
Comprimento da asa & 3,108 & 0,783 & 22,384 & 3,968 & 0,001 \\
Largura da cabeça & 2,036 & 4,291 & 7,660 & 0,475 & 0,635 \\
Largura da face & $-3,671$ & 4,396 & 0,025 & $-0,835$ & 0,404 \\
Cerdas frontais & $-1,472$ & 0,580 & 0,229 & $-2,541$ & 0,011 \\
\hline
\end{tabular}

TABELA 3: Análise por regressão logística múltipla das três características morfométricas de machos de Anastrepha zenildae.

\begin{tabular}{lccccc}
\hline $\begin{array}{c}\text { Constante } \boldsymbol{n} \text { e Variável } \\
\text { independente }\end{array}$ & Coeficiente B & $\begin{array}{c}\text { Erro } \\
\text { Padrão }\end{array}$ & $\begin{array}{c}\text { Relação } \\
\text { Probabilística }\end{array}$ & $\mathbf{z}$ & $\mathbf{p}$ \\
\hline Constante & $-32,887$ & 7,109 & - & $-4,633$ & 0,001 \\
Comprimento do tórax & 6,379 & 1,618 & 589,367 & 3,943 & 0,001 \\
Abs Dif DE & $-1,437$ & 0,573 & 0,238 & 2,506 & 0,012 \\
Comprimento da asa & 3,025 & 0,771 & 20,589 & 3,924 & 0,001 \\
Constante & $-32,887$ & 7,109 & - & $-4,633$ & 0,001 \\
Comprimento do tórax & 6,379 & 1,618 & 589,367 & 3,943 & 0,001 \\
\hline
\end{tabular}




\section{Discussão}

A seleção sexual tem um importante papel na evolução de espécies com sistema de acasalamento em lek. Neste sistema de acasalamento, as fêmeas visitam sítios onde os machos estão agregados defendem um, pequeno território e exibem comportamento de chamada para atrair as fêmeas, estas por sua vez escolheriam o macho mais adequado (BURK; WEBB, 1983; HEADRICK; GOEDEN, 1994). A seleção sexual através da escolha da fêmea pode estar ocorrendo através de características morfológicas. Dentro do gênero Anastrepha, poucos estudos que investigam o papel das características morfológicas na seleção sexual têm sido desenvolvidos (BURK; WEBB, 1983; SCIURANO et al., 2007; SEGURA et al., 2007; SOUZA et al., 2007).

A formação de lek é a estratégia mais frequente no gênero Anastrepha e foi observado em várias espécies dentre elas Anastrepha ludens, A. obliqua (ALUJA et al., 1983; MALAVASI, 1984), A. suspensa (DODSON, 1982), A. fraterculus (MORGANTE et al., 1983), A. sororcula, A. pseudoparalela (TELESDA-SILVA et al., 1985), A. grandis (SILVA, 1991), A. striata (SELIVON et al., 1991; ALUJA et al., 1993) e A. serpentina (ALUJA et al., 1989) e A. zenildae (ALMEIDA et al., 2011). Estudos têm demonstrado uma preferência na escolha feita pelas fêmeas por machos maiores, simétricos ou menos assimétricos que são supostamente mais atraentes, proporcionando uma vantagem seletiva aos seus portadores, resultando no sucesso da cópula (MOLLER; POMIANKOWSKI, 1993). Como consequência, algumas características morfológicas podem estar correlacionadas com sucesso de cópula.

No nosso estudo, observamos que para a maioria das características morfológicas, os machos que obtiveram sucesso de cópula apresentaram em média valores mais altos do que os machos que não obtiveram sucesso de cópula, as variáveis comprimento do tórax, comprimento de asa e o módulo da diferença entre cerdas da placa frontal mostram-se como alvos da seleção sexual em $A$. zenildae, sugerindo que fêmeas podem discriminar os machos por tamanho durante a interação que ocorre no lek.
Dados na literatura corroboram os resultados encontrados na nossa pesquisa; estudos feitos em Anastrepha fraterculus demonstraram que o comprimento de tórax e a largura da asa parecem ser os caracteres morfológicos alvos da seleção sexual, uma vez que estes caracteres se apresentaram fortemente correlacionados com o sucesso de cópula, sugerindo que a diversidade relacionada ao tamanho do corpo pode manter a seleção sexual observada (SCIURANO et al., 2007). Em Anastrepha suspensa, foi verificado que machos maiores são mais propensos a comportamento de chamada e $70 \%$ das fêmeas acasalaram com machos maiores (BURK; WEBB, 1983). Segura et al. (2007), trabalhando com $A$. fraterculus a partir da análise morfométrica, encontraram uma possível associação do comprimento do olho com sucesso de cópula, machos com olhos mais longos, gastam mais tempo realizando o comportamento de corte dentro do lek aumentando chances de cópula, enquanto machos de olhos mais curtos, gastam menos tempo de chamada.

Trabalhos realizados com a mosca-da-fruta Ceratitis capitata indicaram que o tamanho do macho e outras características morfológicas têm relevância no sucesso de cópula. Machos acasalados foram em média maiores do que machos não acasalados e as características morfológicas como comprimento do tórax, comprimento do olho e largura da face são os prováveis alvos da seleção sexual; revelando que comprimento do tórax e olho está positivamente correlacionado com o sucesso da cópula, enquanto largura da face foi negativamente correlacionada com o sucesso da cópula nos machos observados, sugerindo que machos com tórax e olhos maiores e face pequena tem maior sucesso de cópula (RODRIGUERO et al., 2002a; 2002b).

No nosso estudo, o módulo da diferença de cerdas da placa frontal mostrou uma associação com o sucesso de acasalamento, pois os machos mais simétricos acasalaram mais do que os machos não simétricos. Quando a assimetria relativa do número de cerdas da placa frontal e o sucesso de cópula foram analisadas em conjunto, observamos uma diferença significativa na interação dessas variáveis e de uma amostra de 97 machos analisados, a maioria dos indivíduos que obtiveram sucesso de cópula foi simétrica. Estudos 
em dípteros indicam que indivíduos que apresentam assimetria de cerdas podem apresentar redução do sucesso de cópula. Em Drosophila pseudoobscura, machos acasalados eram menos assimétricos do que machos que não acasalaram (MARKOW; RICKER, 1992). Em Ceratitis capitata, Hunt et al. (2004) observaram uma associação entre assimetria e sucesso de acasalamento, machos mais simétricos para cerdas supraorbital tiveram maior sucesso de cópula do que machos assimétricos.

Dentro do gênero Anastrepha, poucos estudos (BURK; WEBB, 1983; SCIURIANO et al., 2007; SEGURA et al., 2007; SOUZA et al., 2007) têm sido realizados enfatizando o papel das características morfológicas na obtenção de parceiros sexuais. Tais estudos podem ser de grande relevância para o desenvolvimento de técnicas de manejo de moscas-dasfrutas, principalmente programas de manejo que utilizam a técnica do macho estéril, uma vez que o conhecimento do comportamento de acasalamento bem como os fatores que determinam a seleção sexual com escolha da fêmea pode ser atribuído a caracteres morfológicos, estando estes caracteres associados ao sucesso ou insucesso de cópula.

\section{Agradecimentos}

A Faculdade de Ciências, Cultura e Extensão do Rio Grande do Norte pelo auxilio com a bolsa de pesquisa. Ao Laboratório de Biologia de Insetos Pragas do Departamento de Biologia Celular e Genética Centro de Biociências da Universidade federal do Rio Grande do Norte, pelo apoio na realização das fotografias e medidas morfométricas.

\section{Referências}

ALUJA, M.; CABRERA, M.; GUILLÉN, J.; CELEDONIO, H. Behavior of Anastrepha ludens, A. obliqua and A. serpentina (Diptera: Tephritidae) on wild mango tree (Mangifera indica) harbouring three McPhail traps. Insect Science and its Application, Cambridge, v. 10, p. 309-318, 1989.

ALUJA, M.; HENDRICHS, M. J.; CABRERA, M. Behavior and interactions between Anastrepha ludens (Loew) and A. obliqua (Macquart) on field cage mango tree I. Leking behavior and male territoriality. In: CARVALLORO, R. (Ed.). Fruit flies of economic importance. 1. ed. Rotterdam: A. A. Balkema, 1983. p. 122-133.
ALUJA, M.; JACOME, G.; BIRKE, A.; LOZADA, N.; QUINTERO, G. Basic patterns of behavior in wild Anastrepha striata (Diptera: Tephritidae) flies under Field-cage conditions. Annals of the Entomological Society of America, Washington, v. 86, n. 6, p. 776-793, 1993.

ALUJA, M.; PINERO, J.; JÁCOME, I.; DIAZ-FLEISCHER, F.; SIVINSK, J. Behaviour of flies in the genus Anastrepha (Trypetinae:Toxotrypanini). In: ALUJA, M; NORBOM, A. L. (Ed.). Fruit flies (Tephritidae): phylogeny and evolution of behavior. 2 ed. Boca Raton: CRC Press, 2000. p. 375-406.

ALMEIDA, L. M.; ARAÚJO, A.; MENDES, N. H. D.; SOUZA, J. M. G. A.; MENEZES, A. A. L. The temporal pattern of mating behavior of the fruit fly, Anastrepha zenildae in the laboratory. Journal of Insect Science, Madison, v. 11, n. 151, p. 1-9, 2011.

ANDERSSON, M. Sexual selection. Princeton: Princeton University Press, 1994. 624 p.

ATCHLEY, W. R.; CHENEY, J. Morphometric differentiation in the viatica group of morabine grasshoppers (Orthoptera: Eumastacidae). Systematic Zoology, Lawrence, v. 23, n. 1, p. 400415, 1974

ARAÚJO, E. L.; MEDEIROS, M. K. M.; SILVA, V. E.; ZUCCHI, R. A. Moscas-das-frutas (Diptera: Tephritidae) no semi-árido do Rio Grande do Norte: plantas hospedeiras e índices de infestação. Neotropical Entomology, Curitiba, v. 34, n. 6, p. 889-894, 2005.

ARAÚJO, E. L.; ZUCCHI, R. A. Moscas-das-frutas (Diptera: Tephritidae) em goiaba Psidium guajava L.), em Mossoró/Assu, RN. Arquivos do Instituto Biológico, São Paulo, v. 1, n. 70, p. 73-77, 2003.

BURK, T.; WEBB, C. Effect of male size on calling propensity, song parameters, and mating success in Caribbean fruit flies, Anastrepha suspensa (Loew) (Diptera: Tephritidae). Annals of the Entomological Society of America, Washington, v. 76, n. 4, p. 678-682, 1983.

CALKINS, C. O.; ASHLEY, T. R. The impact of poor quality of mass-reared Mediterranean fruit flies on the sterile insect technique used for eradication. Journal of Applied Entomology, Goettingen, v. 108, n. 1-5, p. 401-408, 1989.

CLARKE, G. M.; MCKENZIE, J. A. Fluctuating asymmetry as a quality control indicator for insect mass rearing projects. Journal of Economic Entomology, Riverside, v. 85, n. 6, p. 2045-2050, 1992.

DALY, H. V. Insect morphometrics. Annual Review of Entomology, Palo Alto, v. 30, p. 415-438, 1985.

DODSON, G. Mating and territiriality in wild Anastrepha suspensa, (Diptera: Tephritidae) in field cages. Journal of the Georgia Entomological Society, Georgia, v. 17, n. 2, p. 189-200. 1982.

EMLEN, S. T.; ORING, W. L. Ecology, sexual selection, and the evolution of matting systems. Science, Washington, v. 197, n. 4300, p. 215-213, 1977.

GWYNNE, D. T. Courtship feeding increases female reproductive success in bushcrickets. Nature, London, v. 307, n. 5949, p. 361363, 1984.

HEADRICK, D. H.; GOEDEN, R. D. Reproductive behavior of California fruit flies and the classification and evolution of Tephritidae (Diptera) mating systems. Studia Dipterologica, Müncheberg, v. 1, n. 1, p. 194-252, 1994. 
HUNT, M. K.; CREAN, S. C.; WOOD R. J.; GILBURN, A. S. Fluctuating asymmetry and sexual selection in the Mediterranean fruit fly (Diptera, Tephritidae). Biological Journal of the Linnean Society, London, v. 64, n. 3, p. 385-388, 1998.

HUNT, M. K.; NICHOLLS C. J.; WOOD, R. J.; PREDON A. P.; GILBURN, A. S. Sexual selection for symmetrical male medflies (Diptera, Tephritidae) confirmed in the field. Biological Journal of the Linnean Society, London, v. 81, n. 3, p. 347-355, 2004.

JENNIONS, M. D.; PETRIE, M. Variation in mate choice and mating preferences: a review of causes and consequences. Biological Reviews of the Cambridge Philosophical Society, Cambridge, v. 72, n. 2, p. 283-327, 1997.

LUCK, N.; JOLY, D. Sexual selection and mating advantages in the giant sperm species, Drosophila bifurca. Journal of Insect Science, Madison, v. 5, n. 10, p 1-8, 2005.

MALAVASI, A. Estudo de duas espécies crípticas do gênero Anastrepha (Diptera: Tephritidae). 1984. 189 f. Tese (LivreDocência em Biologia) - Universidade de São Paulo, São Paulo. 1984.

MARKOW, T. A.; RICKER, J. P. Male size, developmental stability, and mating success in naturel populations of three Drosophila species. Heredity, Sheffield, v. 69, n. 2, p. 122-127, 1992.

MARTIN, P.; BATESON, P. Measuring behaviour. An introductory guide. Cambridge: Cambridge University Press, 1994. 238 p.

MOLLER, A. P., POMIANKOWSKI, A. Fluctuating asymmetry and sexual selection. Genetica, Dordrecht, v. 89, n. 1, p. 267-269, 1993.

MORGANTE, J. S.; SELIVON, D.; SOLFERINI, V. N., MATIOLI, S. R. Evolutionary patterns in specialist and generalist species of Anastrepha. In: ALUJA, M.; LIEDO, P. (Ed.). Fruit flies: biology and management.1ed. New York: Springer-Verlag, 1993. p. 15-20.

PIZZARI T. Food, vigilance, and sperm: the role of male direct benefits in the evolution of female preference in a polygamous bird. Behavioral Ecology, Oxford, v. 14, n. 14, p. 593-601, 2003.

PROKOPY, R J. Mating behaviour of frugivorous Tephritidae in nature. In: SYMPOSIUM ON FRUIT FLY PROBLEMS, XVI INTERNATIONAL CONGRESS OF ENTOMOLOGY, XVI, 1980, Kyoto. Proceedings... Kyoto: A Ntl. Inst. Agric. Sci. Yatabe, 1980. p. 37-46.

RODRIGUERO, M. S.; VERA, M. T.; RIAL, E.; CAYOL.; J. P.; VILARDI, J. C. Sexual selection on multivariate phenotype in wild and mass-reared Ceratitis capitata (Diptera: Tephritidae). Heredity, Sheffield, v. 89, n. 6, p. 480-487, 2002a.
RODRIGUERO, M. S.; VILARDI, J. C.; VERA, M. T.; CAYOL, J. P.; RIAL, E. Morphometric traits and sexual selection in medfly (Diptera: Tephritidae) under field cage conditions, Florida Entomologist, Lutz, v. 85, n. 1, p. 143-149, 2002 b.

SCIURANO, R.; SEGURA, D.; RODRIGUERO, M.; CENDRA. P. G.; ALLINGHI, A.; CLADERA, J. L.; VILARDI, J. C. Sexual selection on multivariate phenotype in Anastrepha fraterculus (Diptera: Tephritidae) from Argentina. Florida Entomologist, Lutz, v. 90, n. 1, p. 163-170, 2007.

SEGURA, D.; PETIT-MARTY, N.; SCIURANO, R.; VERA, T.; CALCAGNO, G.; ALLINGHI, A.; CENDRA, P. G.; CLADERA, J.; VILARDI, J. Lekking behavior of Anastrepha fraterculus (Diptera: Tephritidae). Florida Entomologist, Lutz, v. 90, n. 1, p. 154-162, 2007.

SIVINSKI, J.; T. BURK. Reproductive and mating behaviour. In: ROBINSON, A. S.; G. HOOPER (Ed.) World Crop Pest. Fruit flies: Their biology, natural enemies and control. 1ed. New York: Elsevier Science, 1989. p. 345-350.

SELIVON, D. ; MORGANTE, J. S. ; SOLFERINI, V. ; MATIOLI, S. Seleção sexual em Anastrepha striata e Anastrepha bistrigata (Diptera: Tephritidae). In: CONGRESSO NACIONAL DE GENETICA, 37, 1991, Caxambu. Caxambu: SBG, 1991. Vol. 14. p. 117.

SILVA, J. G. DA. Biologia e comportamento de Anastrepha grandis (Macquart, 1846) (Diptera:Tephritidae). 1991. $135 \mathrm{f}$. Dissertação (Mestrado em Ciências Biológicas) - Universidade de São Paulo, São Paulo. 1991.

SOUZA, J. M. G. A.; GOUVEIA, M.; PERONDINI, A. L. P.; SELIVON, D. Asymmetry of frontal bristles and postocular setae in species and hybrids of the Anastrepha fraterculus complex (Diptera, Tephritidae). Genetics and Molecular Biology, São Paulo, v. 30, n. 1, p. 145-151, 2007.

TELES-DA-SILVA, M. C.; POLLONI, Y. J.; BRESSAN, S. Mating behavior of some fruit flies of the genus Anastrepha Schiner, 1868 (Diptera:Tephritidae) in laboratory. Revista Brasileira de Entomologia, Curitiba, v. 29, p. 155-164, 1985.

THORNHILL, R. Fluctuating asymmetry and the mating system of the Japanese scorpionfly, Planorpa japonica. Animal Behaviour, St Andrews, v. 44, n. 5, p. 867-879, 1992. 